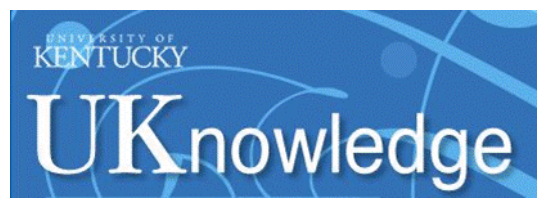

University of Kentucky

UKnowledge

$5-1-2000$

\title{
Infrared Classification of Galactic Objects
}

Željko Ivezić

Princeton University

Moshe Elitzur

University of Kentucky, moshe@pa.uky.edu

Follow this and additional works at: https://uknowledge.uky.edu/physastron_facpub

Part of the Astrophysics and Astronomy Commons, and the Physics Commons

Right click to open a feedback form in a new tab to let us know how this document benefits you.

\section{Repository Citation}

Ivezić, Željko and Elitzur, Moshe, "Infrared Classification of Galactic Objects" (2000). Physics and Astronomy Faculty Publications. 216.

https://uknowledge.uky.edu/physastron_facpub/216

This Article is brought to you for free and open access by the Physics and Astronomy at UKnowledge. It has been accepted for inclusion in Physics and Astronomy Faculty Publications by an authorized administrator of UKnowledge. For more information, please contact UKnowledge@lsv.uky.edu. 


\section{Infrared Classification of Galactic Objects}

Digital Object Identifier (DOI)

http://dx.doi.org/10.1086/312641

\section{Notes/Citation Information}

Published in The Astrophysical Journal Letters, v. 534, no. 1, p. L93-L96.

(c) 2000. The American Astronomical Society. All rights reserved.

The copyright holder has granted permission for posting the article here. 


\title{
INFRARED CLASSIFICATION OF GALACTIC OBJECTS
}

\author{
ŽELJKo IVEZIĆ ${ }^{1}$ AND Moshe ElitzuR ${ }^{2}$ \\ Received 2000 January 28; accepted 2000 March 15; published 2000 April 27
}

\begin{abstract}
Unbiased analysis shows that IRAS data reliably differentiate between the early and late stages of stellar evolution because objects at these stages clearly segregate in infrared color-color diagrams. Structure in these diagrams is primarily controlled by the density distribution of circumstellar dust. The density profile around older objects is the steepest, declining as $r^{-2}$, while young objects have profiles that vary as $r^{-3 / 2}$ and flatter. The different density profiles reflect the different dynamics that govern the different environments. Our analysis also shows that high-mass star formation is strongly concentrated within $\sim 5 \mathrm{kpc}$ around the Galactic center, in support of other studies.
\end{abstract}

Subject headings: catalogs — Galaxy: stellar content — Galaxy: structure — infrared: general — stars: AGB and post-AGB - stars: formation

\section{INTRODUCTION}

The IRAS all-sky survey provides a unique opportunity to classify the infrared properties of astronomical objects from a homogeneous data set obtained with a single facility. Crosscorrelations of various catalogs with the IRAS Point Source Catalog (PSC) showed that certain Galactic objects tend to cluster in well-defined regions of IRAS color-color diagrams. Notable examples include H II regions (e.g., Hughes \& MacLeod 1989; Wood \& Churchwell 1989, hereafter WC) and asymptotic giant branch (AGB) stars (van der Veen \& Habing 1988, hereafter $\mathrm{VH}$ ). The reason for this clustering was not understood, nor was it clear whether such biased analysis based on preselection implies reliable selection criteria. From detailed modeling of dusty winds, we were able to validate the $\mathrm{VH}$ selection criterion proposed for AGB stars and to explain its origin (Ivezić \& Elitzur 1995, hereafter IE95). Here we extend this approach to all Galactic PSC sources in an unbiased analysis of IRAS fluxes without prior selections.

\section{IRAS DATA AND ITS CLASSES}

There are 6338 PSC sources with flux quality of at least 2 in all four IRAS channels. Removing the 651 sources identified as extragalactic (Beichman et al. 1985) produces our basic data set of 5687 Galactic IR objects. For AGB stars, we have shown (IE95) that a high IRAS quality does not guarantee that the flux is intrinsic to the point source itself; high-quality 60 and $100 \mu \mathrm{m}$ PSC fluxes can have a cirrus origin instead. Figure 1 shows that the problem afflicts all sources, not just AGB stars. Its $x$-axis is cirr $3 / F_{60}$, a measure of the ratio of cirrus background noise to the $60 \mu \mathrm{m}$ signal. Intrinsic fluxes should have nothing to do with background emission, yet the [100-60] color is strongly correlated with cirr $3 / F_{60}$ when this noise indicator exceeds a certain threshold. The PSC fluxes of sources above this threshold reflect cirrus, not intrinsic emission. Following IE95, we remove all sources with cirr3 $>2 F_{60}$ to eliminate cirrus contamination. This leaves 1493 objects that can be considered a reliable representation of Galactic infrared point sources.

\footnotetext{
${ }^{1}$ Princeton University, Department of Astrophysical Sciences, Princeton, NJ 08544-1001; ivezic@astro.princeton.edu.

${ }^{2}$ Department of Physics and Astronomy, University of Kentucky, Lexington, KY 40506-0055; moshe@pa.uky.edu.
}

We submitted these data to the program AutoClass for an unbiased search of possible structure. AutoClass employs Bayesian probability analysis to automatically abstract a given database into classes (Goebel et al. 1989). AutoClass detected that the PSC sources belong to four distinct classes that occupy separate regions in the four-dimensional space spanned by IRAS fluxes. Figure 2 (left) shows the two-dimensional projection of this space onto the [100-60]-[25-12] color plane. The IRAS colors of blackbody emission, which are independent of the blackbody temperature as long as it exceeds $\sim 700 \mathrm{~K}$, are marked by the large dot. There is hardly any data at that point. Instead, the data are spread far away from it, indicating that $I R A S$ fluxes are dominated by surrounding dust. But rather than random scatter, the data show clear structure, with the four AutoClass classes occupying well-defined color regions. As a further check on the reality of these classes, we constructed their Galactic distributions (shown in Fig. 2, right).

Classes A and B clearly separate in the color-color diagram. Classes $\mathrm{C}$ and $\mathrm{D}$, on the other hand, are mixed together and are distinguished by their flux levels: class $\mathrm{C}$ fluxes are typically 10-100 times higher in all four bands. In principle, a single family could produce such behavior if split into nearby and distant objects, but this is not the case here. Extragalactic and heliocentric selection effects are ruled out by the Galactic distributions. Both classes are composed of Galactic disk objects, but the median flux of class $\mathrm{C}$ is 16 times higher. If the two were drawn from the same population, class $C$ sources would be on the average 4 times closer and their Galactic latitude distribution 4 times wider. Instead, the latitude histograms are essentially the same; both are centered on $b=0^{\circ}$ with FWHM $1.5 \pm 0.3$ for class $C$ and $1.5 \pm 0.1$ for class D. Class D fluxes vary by more than $10^{3}$, consistent with a population distributed throughout the entire Galactic plane. ${ }^{3}$ In contrast, class $\mathrm{C}$ fluxes vary by a factor of only $\sim 20$ and exceed $10^{3} \mathrm{Jy}$ at both 60 and $100 \mu \mathrm{m}$ for all sources. This is consistent only with a population confined to, at most, $\sim 5 \mathrm{kpc}$ around the Galactic center with a minimal luminosity of $10^{3} L_{\odot}$. The Galactic longitude distributions corroborate these conclusions. Although both class $\mathrm{C}$ and D sources channel most of their luminosity to the farIR, their spatial and luminosity distributions are very different.

${ }^{3}$ Class A fluxes have a similar dynamic range. 


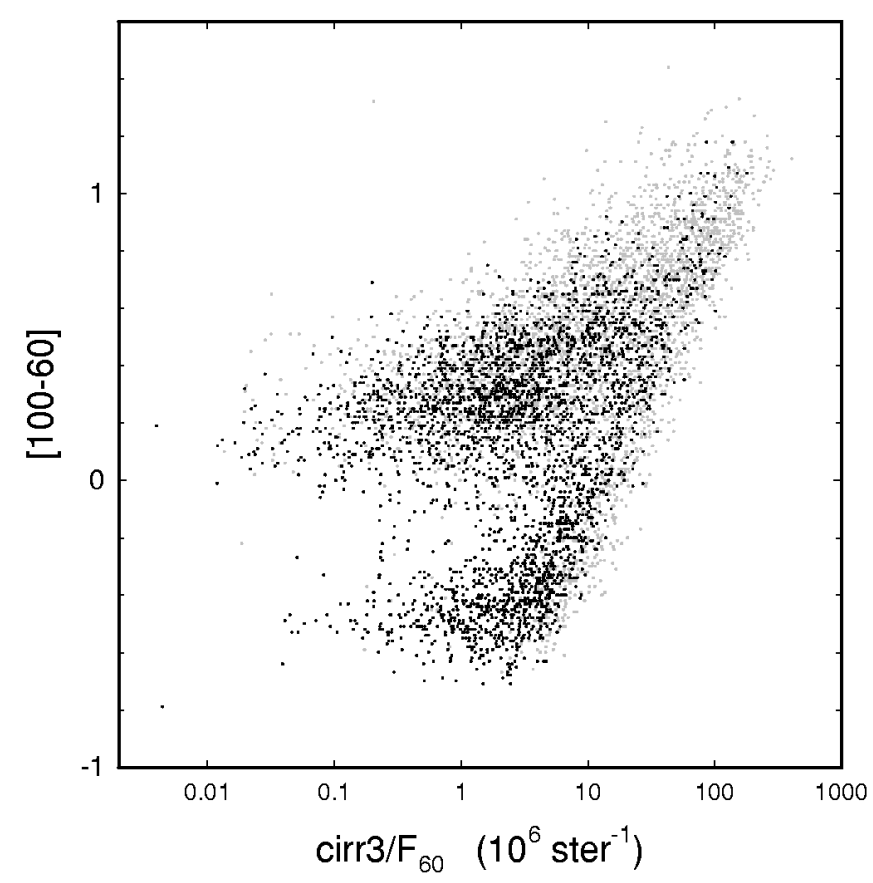

FIG. 1.-The [100-60] color vs. cirr $3 / F_{60}$, where $F_{60}$ is the $60 \mu \mathrm{m} I R A S$ flux and cirr3 is the IRAS surface brightness at $100 \mu \mathrm{m}$ around the point source. The flux quality for all sources is at least 2 in all four IRAS bands, and 3 for those marked with dark dots. In spite of the high flux quality, the strong correlation with the cirrus emission at large values of cirr $3 / F_{60}$ is evident.

\section{THEORY}

In IE95 we identified the cause of the particular IRAS colors of AGB stars: the dusty wind problem possesses general scaling properties. For a given dust composition, the solutions are predominantly determined by a single input parameter-the overall optical depth. All other input is largely irrelevant. As a result, the solutions occupy well-defined regions in color-color diagrams. Indeed, the IRAS measured colors of AGB stars fall in the regions outlined by wind solutions with visual optical depth $\tau_{V} \lesssim 100$.

In Ivezić \& Elitzur (1997, hereafter IE97), we showed that scaling is a general property of radiatively heated dust under all circumstances, not just in AGB winds. The most general dust radiative transfer problem contains only two input quantities whose magnitudes matter: $\tau_{V}$ and the dust temperature $T_{1}$ at some point. All other input is defined by dimensionless, normalized profiles that describe (1) the spectral shape of the external radiation, (2) the spectral shape of the dust absorption and scattering coefficients, and (3) the dust spatial distribution. Physical dimensions such as, e.g., luminosity and linear sizes are irrelevant. Scaling applies to arbitrary geometries, and in IE97 we conducted extensive numerical studies of its consequences for spherical shells. With a blackbody spectral shape for the heating radiation, the temperature $T$ hardly affects IRAS colors when $T>2000 \mathrm{~K}$. The reason is that $T$ is much higher than the Planck equivalent of the shortest IRAS wavelength $(12 \mu \mathrm{m})$, which is only $240 \mathrm{~K}$. Although this result was obtained in spherical solutions, its validity is general. Since neither its luminosity nor spectral shape matter, the heating source is quite irrelevant for the IRAS colors. Different objects segregate in IRAS color-color diagrams not because they have different central sources, but rather because their dust shells are different.
In our extensive study of spherical shells, we examined also the effect of $T_{1}$, which was selected as the dust temperature on the shell inner boundary. As with the heating radiation, and for the same reasons, $T_{1}$ hardly affects IRAS colors when varied over the plausible range of dust sublimation temperatures 700-2000 K (IE97). This leaves the dust's composition, optical depth, and spatial distribution as the only potentially significant reasons for the variation of IRAS colors among different families of Galactic objects. However, dust properties generally do not show large variations among different objects and thus cannot be expected to induce substantial variations in infrared emission. The same applies to $\tau_{V}$, whose range of values is similar in most families. We conclude that objects segregate in IRAS color-color diagrams primarily because their spatial dust distributions are different.

We corroborate these conclusions in detailed modeling with the code DUSTY (Ivezić, Nenkova, \& Elitzur 1999). In these calculations, a point source emitting as a blackbody with temperature $5000 \mathrm{~K}$ is surrounded by dust whose absorption and scattering coefficients are those of standard interstellar mix. The dust is distributed in a spherical shell whose temperature on the inner boundary is $T_{1}=1000 \mathrm{~K}$. The shell density profile is taken as a power law $r^{-p}$, with $p$ a free parameter. Scaling ensures that the solution set for each $p$ is a one-parameter family characterized by $\tau_{V}$, leading to the distinct tracks shown in the [60-25]-[25-12] color-color diagram in Figure 3. These tracks properly delineate the distribution of IRAS sources, and we have verified that the same applies also to the [100-60] colors shown in Figure 2. Tracks for different $p$ 's differ in the colorcolor regions that they cross and in the distance induced by $\tau_{V}$ variation; the steeper the density profile, the smaller the distance along the track for the same change in $\tau_{V}$. Both properties arise from the differences in relative amounts of material placed at different temperatures, and both are reflected in the data. Class A sources are well explained by the $p=2$ track; indeed, they cluster close to the blackbody point. In contrast, classes $\mathrm{C}$ and $\mathrm{D}$ are explained by tracks of much flatter density distributions and are located rather far from the blackbody colors; it takes only $\tau_{V} \sim 0.1$ to move an object along these tracks all the way from the blackbody point to the region populated by IRAS sources.

Class B cannot be reasonably explained by the same models, even though its colors can be produced by extending the $p=$ 2 track beyond $\tau_{V}=100$. In addition to the implausible optical depths this requires, class B is unlikely to be the high- $\tau_{V}$ end of class A given their different Galactic distributions. An alternative to $\tau_{V}$ variation is to modify colors by removal of hot dust. The displayed tracks have $T_{1}=1000 \mathrm{~K}$, as appropriate for shells whose inner boundaries are controlled by dust sublimation, but in detached shells $T_{1}$ is both lower and arbitrary. The dashed-line tracks in Figure 3 show the effect of lowering $T_{1}$ on the $p=2$ track. The difference between $T_{1}=1000$ and $300 \mathrm{~K}$ is marginal because both are higher than the Planck equivalent of $12 \mu \mathrm{m}$. However, further reduction in $T_{1}$ alters the track significantly, adequately explaining class B colors.

\section{DISCUSSION}

Our modeling confirms that the primary reason for different IRAS classes is different dust density distributions. Only 5\% of the sources require an additional variation of the dust temperature on the shell inner boundary. Spherical shells were employed here as the simplest method to model extended, 

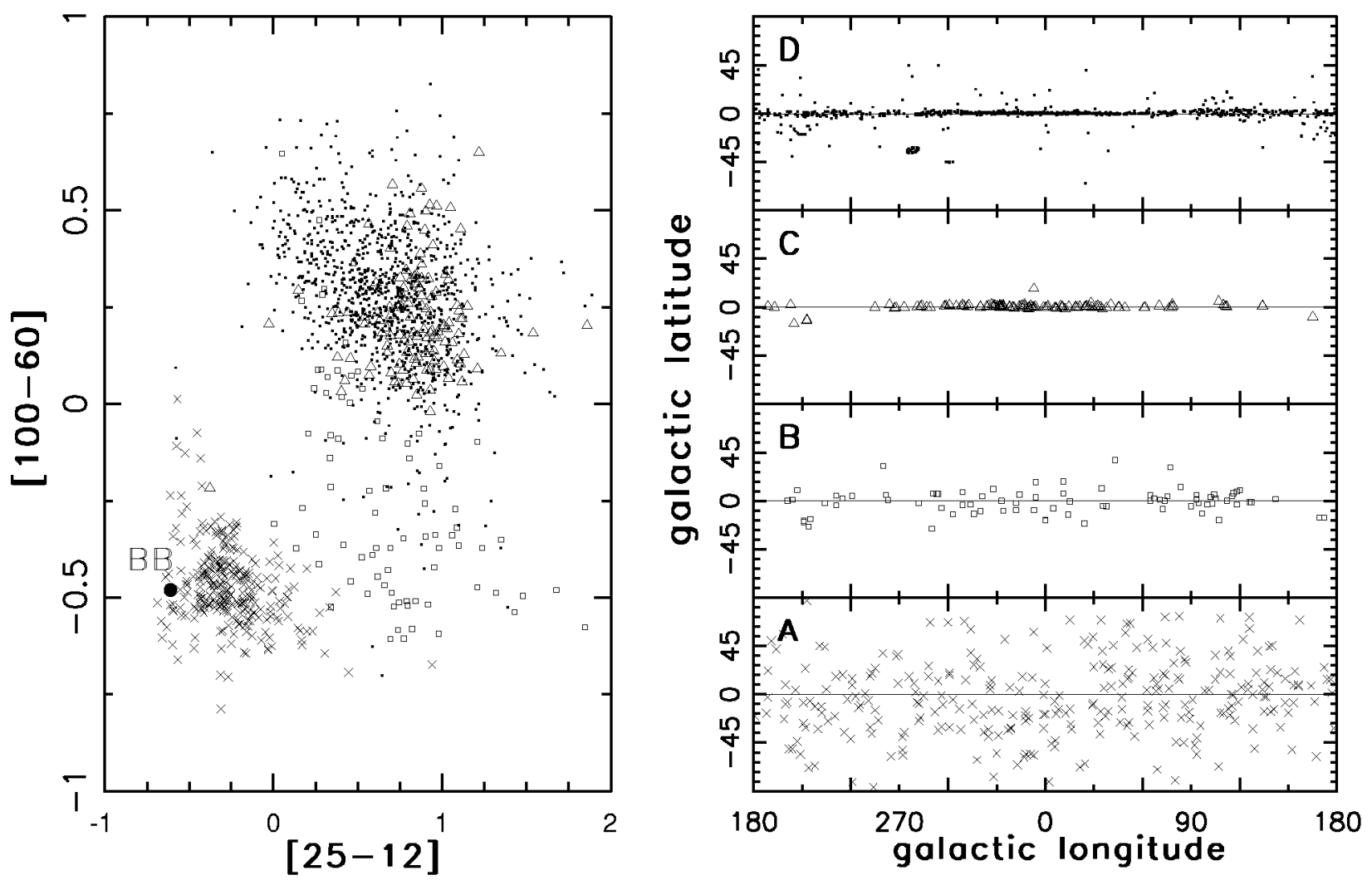

FIG. 2.-Left: [100-60]-[25-12] color-color diagram for all the Galactic IRAS sources free from cirrus contamination. The four classes identified by AutoClass are marked with different symbols: Class A (282 sources), crosses; B (80 sources), squares; C (119 sources), triangles; D (1013 sources), dots. The large dot marked BB denotes the colors of blackbody emission with temperature $T>700 \mathrm{~K}$. Right: Galactic distributions of the four classes.

three-dimensional dust distributions. Power laws were used just for illustration purposes, and although their actual values should not be taken literally, they provide a good indication of the overall behavior of the density distributions in the four IRAS classes. The presence of disks, expected in various sources, should not significantly affect our conclusions. The standard disk spectrum $F_{\nu} \propto \nu^{1 / 3}$ produces a single point in color-color diagrams and thus cannot effect the observed scatter. This spectrum is modified if the disk is embedded in an extended shell, but then the disk is expected to dominate only at submillimeter and millimeter wavelengths, longer than those observed by IRAS (Miroshnichenko et al. 1999). Flared disk emission can be shown equivalent to that from a flat disk embedded in an appropriate spherical shell. Therefore, such a configuration cannot modify our conclusions, either.

We have queried the SIMBAD database about our sample sources. SIMBAD identifications are occasionally ambiguous ("maser") and sometimes less than informative ("part of cloud"), and reliability is not always certain. Nevertheless, they provide useful clues when there are major trends in the data. Class A returned the most decisive results- $88 \%$ of its members have possible optical identifications, of which roughly $90 \%$ are commensurate with AGB stars. Since class A obeys the VH criterion, this corroborates our earlier finding (IE95) that this criterion is both sufficient and necessary for AGB selection. In IE95 we present a thorough analysis of the IRAS fluxes of AGB stars, including color tracks geared specifically for these objects (single chemistry grains, appropriate stellar temperature, etc.). While that analysis was necessary to verify the $\mathrm{VH}$ criterion, the $p=2$ track presented here captures the essence of the more detailed study, demonstrating that the density distribution is the leading factor in controlling IRAS colors. These circumstellar shells have the steepest density distribution, setting them apart in the color-color diagrams. In addition, the color tracks are primarily controlled by a single parameter, $\tau_{V}$, hence the compactness of this class color region.

Class B had the same identification rate, but its composition is not as homogeneous. Planetary nebulae constitute $40 \%$ of positive and possible identifications. At 13\%, the only other significant group is "emission-line stars," a classification consistent with planetary and reflection nebulae. The remaining identifications span a variety of objects, indicating that detached shells may occasionally form under different circumstances. Significantly, the optical depths required for class B are lower than for the others; the dashed-line tracks in Figure 3 terminate at $\tau_{V}=10$.

SIMBAD identification rates for the two other classes are much lower-only $32 \%$ for class $\mathrm{C}$ and $21 \%$ for D. Among the 38 class $\mathrm{C}$ identifications, $\mathrm{H}$ II regions constitute the only significant group with 15 (40\%). In class D, too, $\mathrm{H}$ II regions constitute the single largest group with $23 \%$ of the identifications, followed by young stellar objects at $11 \%$ and planetary nebulae at $10 \%$. These two classes are clearly dominated by star formation and early stages of stellar evolution, in agreement 


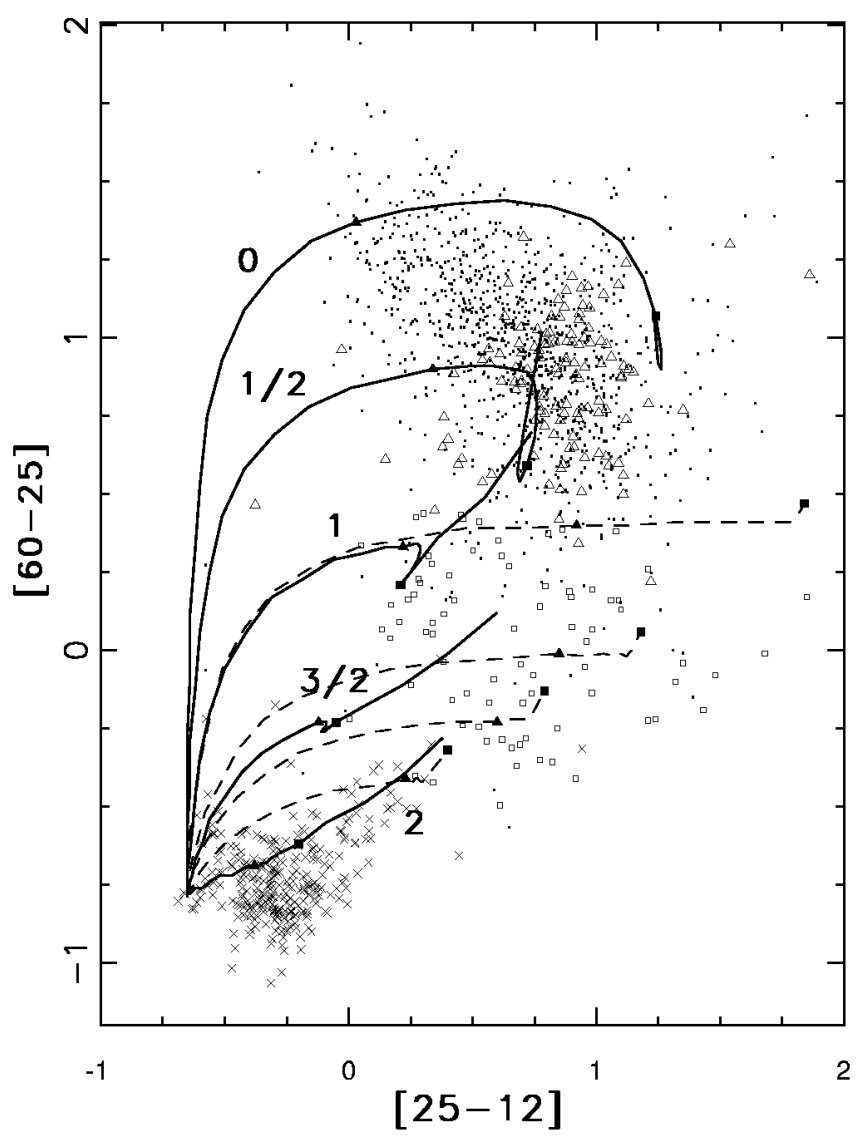

FIG. 3.-The [60-25]-[25-12] color-color diagram. Data are for the same sources presented in Fig. 2 using the same symbols. The full lines are model tracks for spherical dust shells with $T_{1}=1000 \mathrm{~K}$ on their inner boundary and $r^{-p}$ density profiles with $p=0, \frac{1}{2}, 1, \frac{3}{2}$ and 2 , as marked. The position along each track increases with dust optical depth $\tau_{V}$ away from the common origin $\tau_{V}=0$ at the blackbody colors, with $\tau_{V}=0.1$ marked by a filled triangle and $\tau_{V}=10$ by a filled square. The end point for each track is $\tau_{V}=100$. Dashedline tracks show the effect on the $p=2$ track of lowering $T_{1}$ to 300 (lowermost track), 200, 150, and $100 \mathrm{~K}$ (uppermost track). These tracks end at $\tau_{V}=10$.

with their Galactic distributions and with previous attempts to associate star-forming regions with IRAS colors. In the most extensive study of this kind, $83 \%$ of 1302 IRAS-selected sources were found to be embedded in molecular clouds and thus trace star formation, and the selection criteria ([25$12] \geq 0,[60-25] \geq 0.4)$ essentially identify classes $C$ and $D$ (Wouterloot \& Brand 1989; Wouterloot et al. 1990).
The IRAS colors of classes C and D imply dust density distributions flatter than for AGB stars. These colors are spread over large regions, reflecting variation in density profiles in addition to optical depth. The spread in [100-60] colors is smaller than in [60-25] because all shells are optically thin at both 100 and $60 \mu \mathrm{m}$, while their $25 \mu \mathrm{m}$ optical depth can become significant (IE97). Class C colors occupy a subregion of class $\mathrm{D}$ and are produced by the optically thick end $\left(\tau_{V} \gtrsim 1\right)$ of flat density distributions. Objects whose colors fall in that region can belong to either class $\mathrm{C}$ or $\mathrm{D}$. However, among all sources with class D colors, those with high fluxes $(>1000 \mathrm{Jy}$ at both 60 and $100 \mu \mathrm{m}$ ) concentrate in a compact color region, hence the separate class $C$. Since all class $C$ sources have $L>10^{3} L_{\odot}$, they are high-mass objects and their concentration in the inner $\sim 5 \mathrm{kpc}$ of the Galaxy shows that the high-mass star formation rate decreases with distance from the Galactic center. This result is in agreement with studies of star formation inside and outside the solar circle (Wouterloot et al. 1995; Casassus et al. 2000).

Our results explain the findings of all earlier studies that were based on object preselection and reveal the limitations of that approach. By example, consider the WC study. After identifying IRAS counterparts of known ultracompact $\mathrm{H}$ II regions, WC proposed the corresponding colors as a necessary and sufficient selection for all ultracompact $\mathrm{H}$ II regions and proceeded to estimate the birthrate of $\mathrm{O}$ stars. Codella, Felli, \& Natale (1994) then found that most H II regions in a more extended catalog indeed obeyed the WC color criterion. However, that also included diffuse-not just compact-H II regions, so WC overestimated the $\mathrm{O}$-star birthrate. This clearly demonstrates the shortcomings of any classification based on a preselected population. The unbiased analysis presented here shows that IRAS colors reflect primarily the dust density profiles of circumstellar shells and provide a unique indication of the underlying object only in the case of AGB stars. IRAS data in itself is sufficient for differentiating young and old stellar objects; apart from a limited number of detached shells, IRAS sources belong to two distinct groups as is evident from both the color-color diagrams and the Galactic distributions: (1) class A sources are at the late stages of stellar evolution and (2) class $\mathrm{C}$ and $\mathrm{D}$ sources are objects at the early evolutionary stages. This differentiation occurs because the density distributions of dust around young stellar objects have flatter profiles, reflecting the different dynamics that govern the different environments.

Support by NASA and NSF is gratefully acknowledged.

\section{REFERENCES}

Beichman, C. A., Neugebauer, G., Habing, H. J., Clegg, P. E., \& Chester, T. J. 1985, IRAS Catalogs and Atlases (Washington, DC: US GPO)

Casassus, S., Bronfman, L., May, J., \& Nyman, L.-Å. 2000, A\&A, in press (astro-ph/9912340)

Codella, C., Felli, M., \& Natale, V. 1994, A\&A, 284, 233

Goebel, J., et al. 1989, A\&A, 222, L5

Hughes, V. A., \& MacLeod, G. C. 1989, AJ, 97, 786

Ivezić, Ž., \& Elitzur, M. 1995, ApJ, 445, 415 (IE95) 1997, MNRAS, 287, 799 (IE97)

Ivezić, Ž., Nenkova, M., \& Elitzur, M. 1999, User Manual for DUSTY, University of Kentucky Internal Report
Miroshnichenko, A., Ivezić, Ž., Vinković, D., \& Elitzur, M. 1999, ApJ, 520, L115

van der Veen, W. E. C. J., \& Habing, H. J. 1988, A\&A, 194, 125 (VH)

Wood, D. O. S., \& Churchwell, E. 1989, ApJ, 340, 265 (WC)

Wouterloot, J. G. A., \& Brand, J. 1989, A\&AS, 80, 149

Wouterloot, J. G. A., Brand, J., Burton, W. B., \& Kwee, K. K. 1990, A\&A, 230, 21

Wouterloot, J. G. A., Fiegle, K., Brand, J., \& Winnewisser, G. 1995, A\&A, 301,236 